\title{
Triboelectric Generators for Sustainable Reduction Leading to Nanoparticles and Nanoclusters
}

Vishal Kumar, ${ }^{\dagger}$ Pillalamarri Srikrishnarka,$\dagger$ Jyoti Sarita Mohanty, ${ }^{\dagger}$ Murugesan Paulthangam

Kannan, ${ }^{\dagger}$ Ramamurthy Nagarajan, ${ }^{\ddagger}$ Thalappil Pradeep ${ }^{\dagger *}$

†Department of Chemistry, DST Unit of Nanoscience (DST UNS) and Thematic Unit of Excellence (TUE), Indian Institute of Technology Madras, Chennai 600036, India

$\neq$ Department of chemical Engineering, Indian Institute of Technology Madras, Chennai 600036, India

Number of pages: 10

Number of figures: 6

Number of tables: 1

*Corresponding author: Thalappil Pradeep, Department of Chemistry, DST Unit of Nanoscience (DST UNS) and Thematic Unit of Excellence (TUE), Indian Institute of Technology Madras, Chennai 600036, India

E-mail: pradeep@iitm.ac.in 
Table of Contents

\begin{tabular}{|l|l|l|}
\hline \multicolumn{1}{|c|}{ No. } & \multicolumn{1}{|c|}{ Description } & Page No. \\
\hline 1. & Figure S1. Peak current at different separation between Al electrodes. & S3 \\
\hline 2. & Table S1. A brief comparison of performance of published TENGs with & S4 \\
\hline 3. & our TG. & \\
\hline solution. & Figure S2. Current and voltage characteristics for aqueous gold & S5 \\
\hline 4. & Figure S3. Current and voltage characteristics for gold solution in & S6 \\
\hline 5. & Figure S6. UV-Vis spectra for MSA-AuNPs and GSH-AuNPs. & S9 \\
\hline 5. & Figure S4. XPS spectrum of MUA-AuNCs in the Au 4f region. & S7 \\
\hline
\end{tabular}




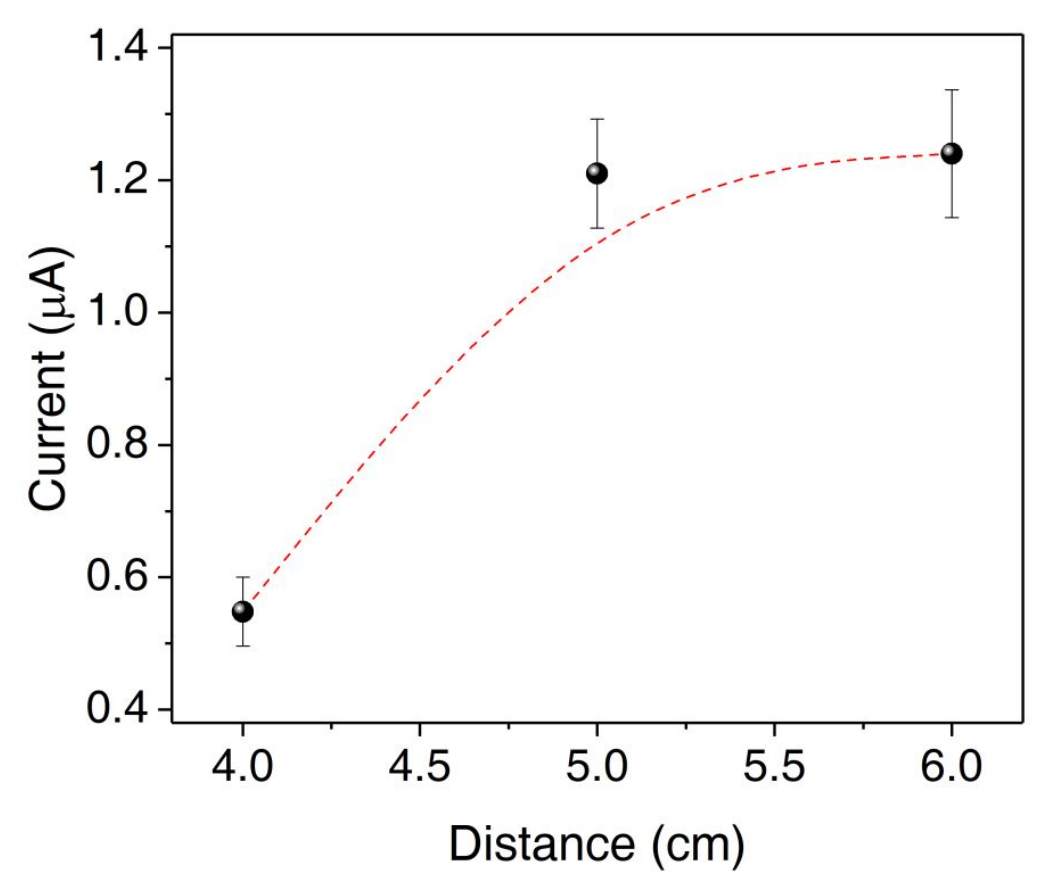

Figure S1. Peak current at different separation between Al electrodes. 
Typically, in TENG, nanostructured materials are used to increase the effective contact area, which significantly amplifies the electrostatic charging compared to normal surface. The effect of this increased charging is reflected mainly as a high-voltage output, but the increase in current in not as substantial. Since power $(P=I \times V)$, the power values are much higher in such systems. But high voltage $(>100 \mathrm{~V})$ in such systems might have a negative effect on the synthesis of NPs and NCs.

Table S1. A brief comparison of performance of published TENGs with our TG.

\begin{tabular}{|l|l|l|l|l|l|}
\hline & Voltage & Current & Power & Mode & Materials \\
\hline This work & $1.4 \mathrm{~V}$ & $1.2 \mu \mathrm{A}$ & $\begin{array}{l}1.45 \mu \mathrm{W}(\sim 1 \\
\mathrm{M} \Omega)\end{array}$ & sliding & 1 . Teflon sheet \\
2. paper
\end{tabular}

*The values of voltage and current represent the value at peak power. 

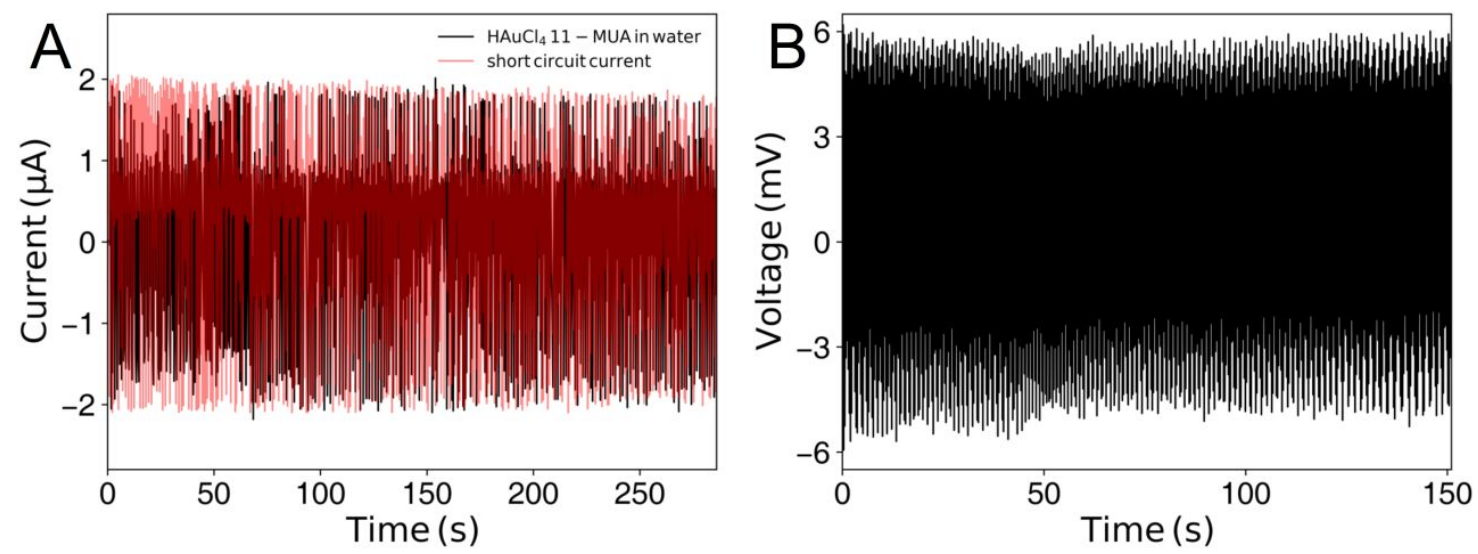

Figure S2. (A) Short circuit current for TG (red), and the current (black) drawn and (B) voltage for the aqueous gold solution during synthesis of MUA-AuNPs. 

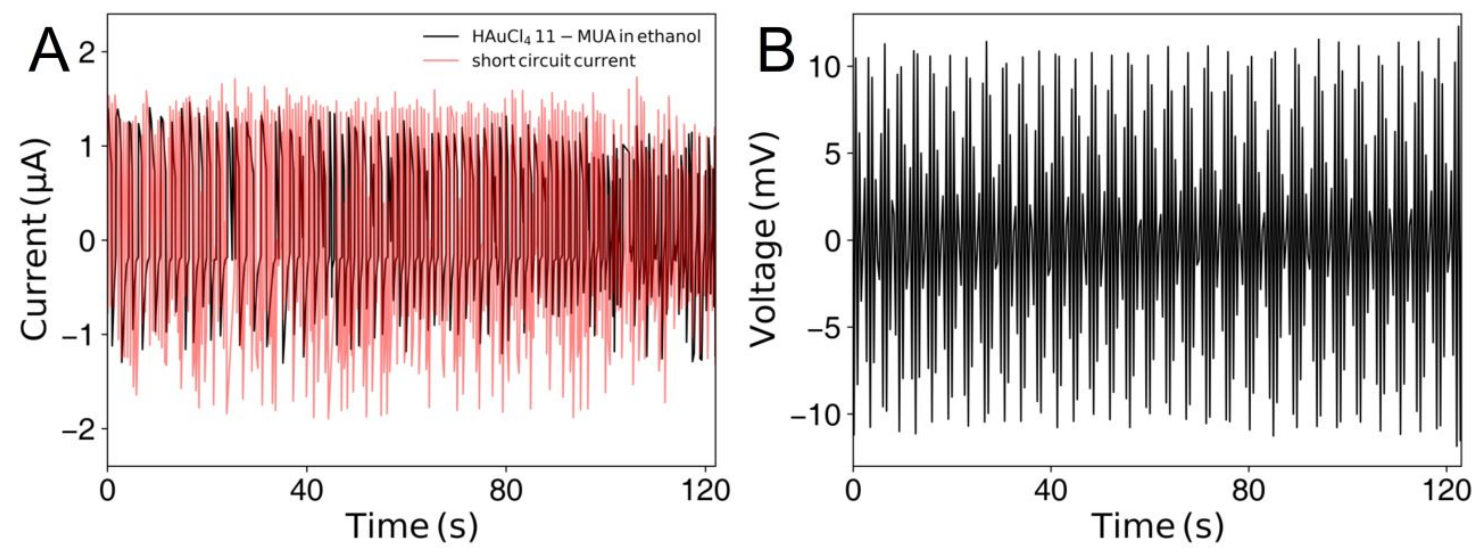

Figure S3. (A) Short circuit current for TG (red) and the current (black) drawn, and (B) voltage for the gold solution in ethanol, during synthesis of MUA-AuNCs. 


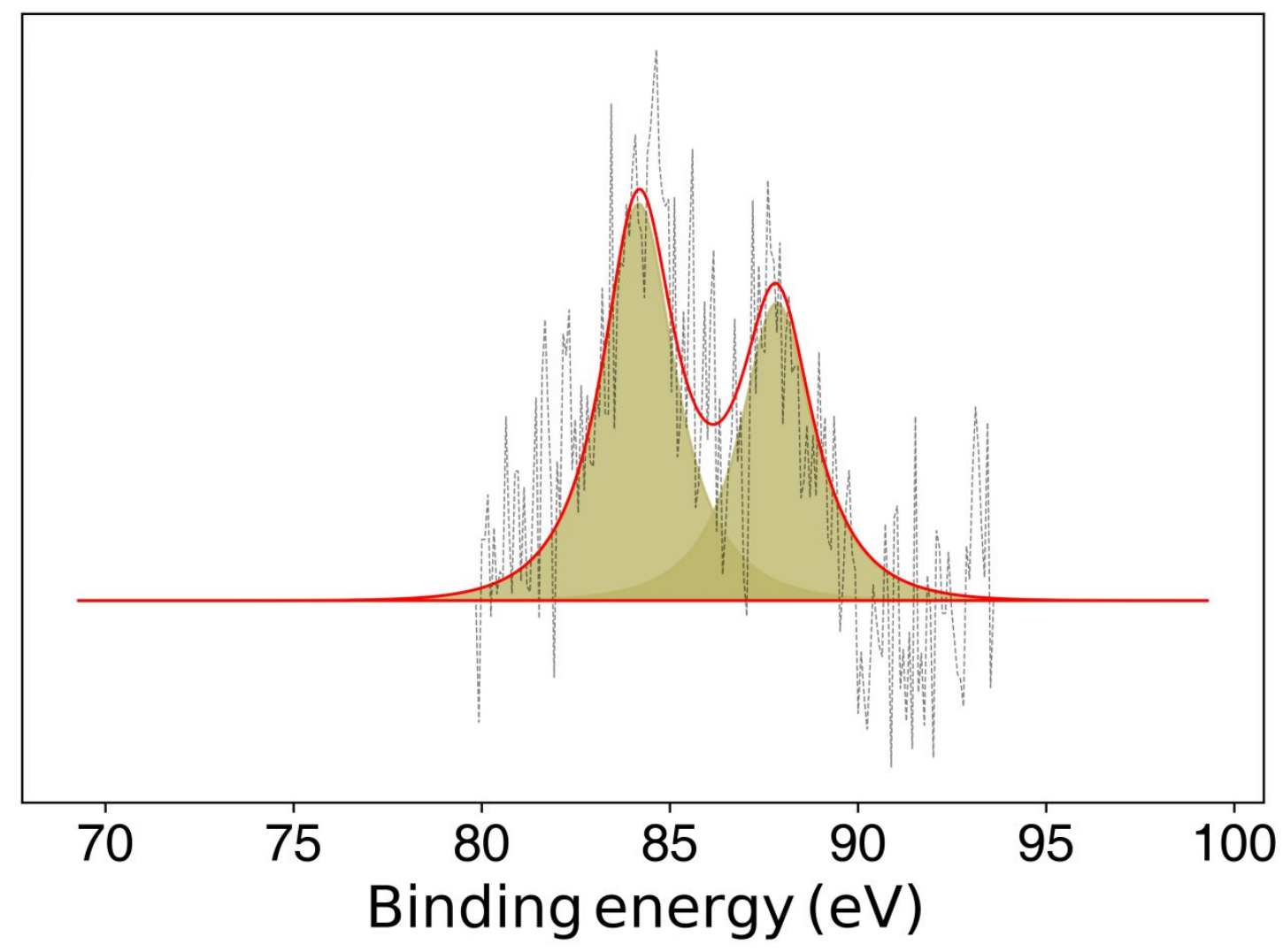

Figure S4. XPS spectrum of MUA-AuNCs in the Au 4f region. 


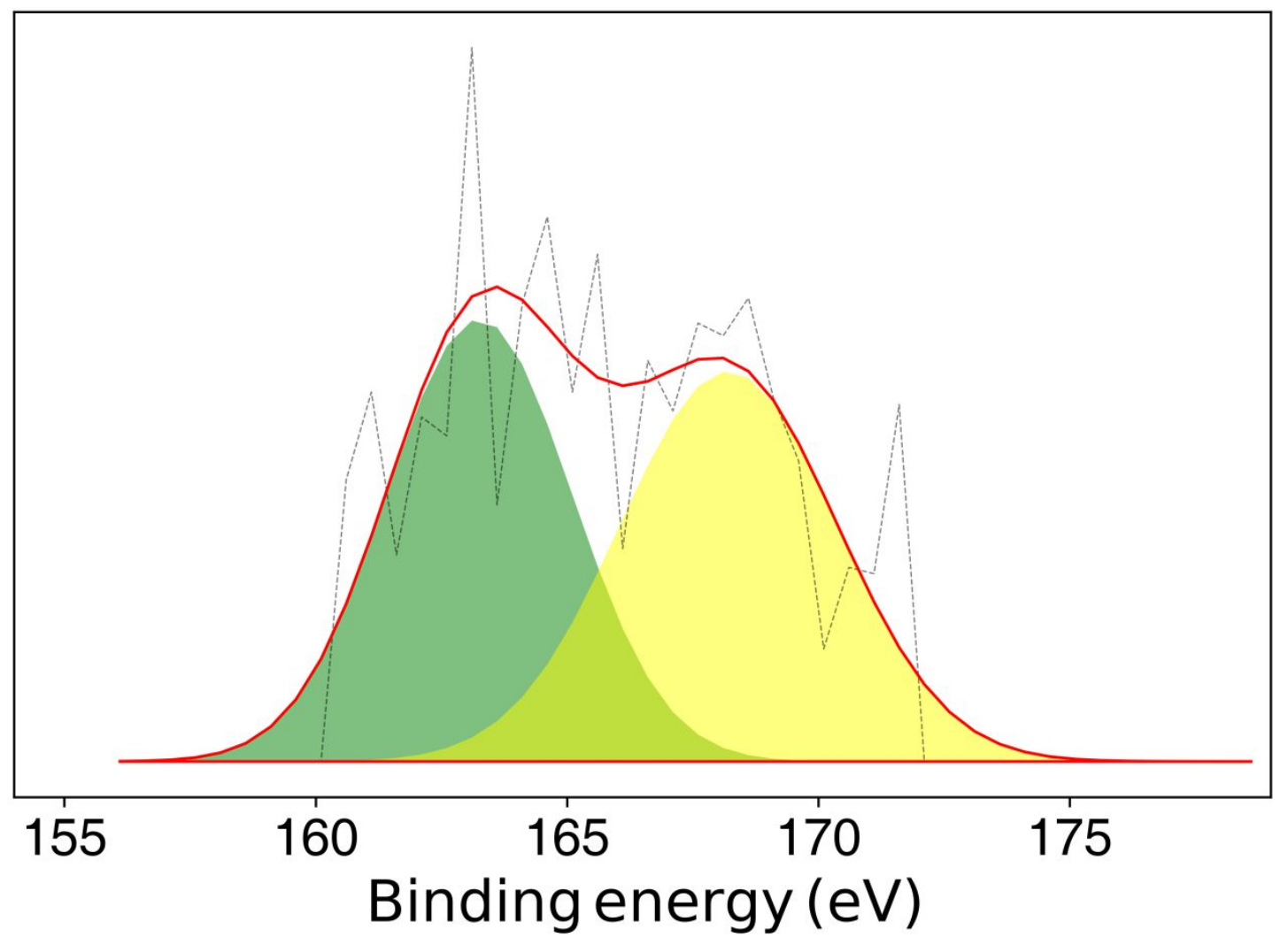

Figure S5. XPS spectrum of MUA-AuNCs (fitted using the survey spectra) in the S $2 p$ region. 

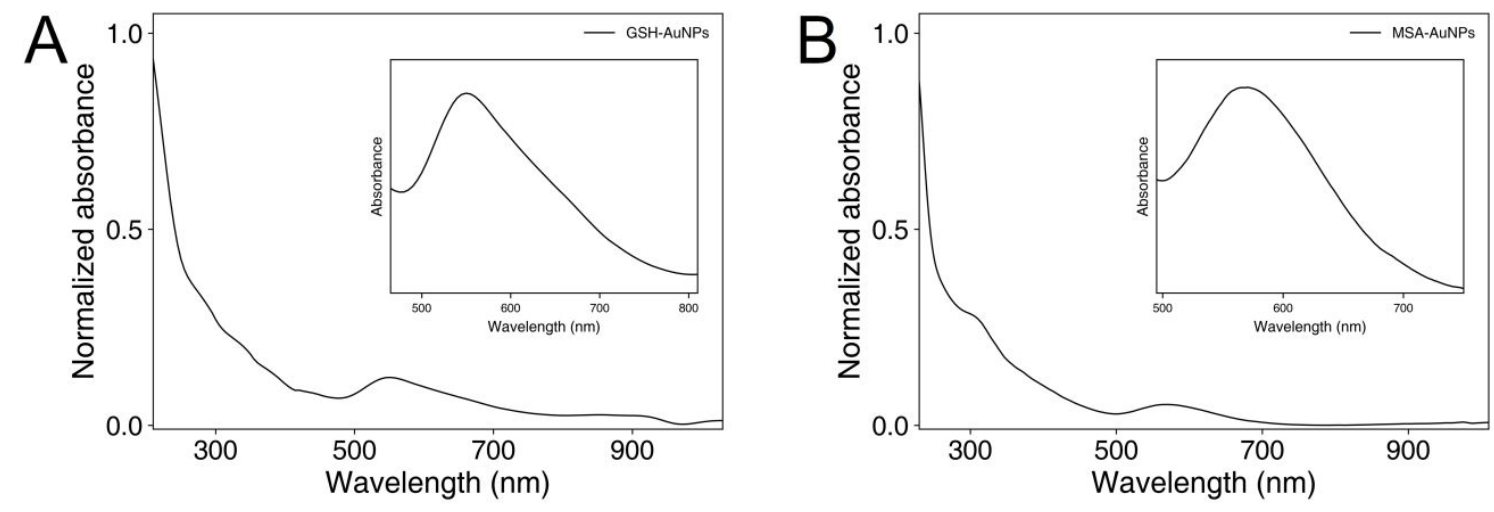

Figure S6. UV-Vis spectra for (A) GSH-AuNPs, and (B) MSA-AuNPs and inset showing the plasmonic peak at $550 \mathrm{~nm}$ and $560 \mathrm{~nm}$, respectively. 


\section{REFERENCES}

(1) Zhong, J.; Zhong, Q.; Fan, F.; Zhang, Y.; Wang, S.; Hu, B.; Wang, Z. L.; Zhou, J. Finger Typing Driven Triboelectric Nanogenerator and Its Use for Instantaneously Lighting up LEDs. Nano Energy 2013, 2 (4), 491-497, DOI 10.1016/j.nanoen.2012.11.015.

(2) Zhu, Z.; Xia, K.; Xu, Z.; Lou, H.; Zhang, H. Starch Paper-Based Triboelectric Nanogenerator for Human Perspiration Sensing. Nanoscale Research Letters 2018, 13 (1), 365, DOI 10.1186/s11671-018-2786-9.

(3) Zheng, Y.; Cheng, L.; Yuan, M.; Wang, Z.; Zhang, L.; Qin, Y.; Jing, T. An Electrospun Nanowire-Based Triboelectric Nanogenerator and Its Application in a Fully Self-Powered UV Detector. Nanoscale 2014, 6 (14), 7842-7846, DOI 10.1039/C4NR01934B. 